\title{
A Comparison of Entanglement Measures
}

\author{
Jens Eisert ${ }^{1}$ and Martin B. Plenio ${ }^{2}$ \\ ${ }^{1}$ Institut für Physik, Universität Potsdam, Germany \\ ${ }^{2}$ Blackett Laboratory, Imperial College, Prince Consort Road, London SW7 2BZ, U.K.
}

\begin{abstract}
We compare the entanglement of formation with a measure defined as the modulus of the negative eigenvalue of the partial transpose. In particular we investigate whether both measures give the same ordering of density operators with respect to the amount of entanglement.
\end{abstract}

PACS-numbers: 03.67.-a, 03.65.Bz

\section{INTRODUCTION}

Entanglement is a key property that makes quantum information theory different from its classical counterpart [1]. Maximally entangled states, for example, are the basis of quantum state teleportation which has recently been demonstrated [2, 4 . Under realistic conditions, however, one will only be able to generate partially entangled mixed states. It is then of interest to be able quantify the amount of entanglement in such states. Some measures of entanglement for mixed states have been suggested recently [5 8 . They are useful, for example, as upper bounds to entanglement purification protocols $19-13$ and to the quantum channel capacity of certain quantum communication channels [5, 8, 14].

Unfortunately entanglement measures for mixed states (which are relevant in the presence of noise) are usually quite hard to calculate analytically, although an analytic expression for the entanglement of formation of two spin$1 / 2$ particles is now known [15]. The general case remains unsolved. For some problems, however, it is not so important to know the exact amount of entanglement (a quantity that is not unique anyway). It would be completely sufficient if one knew which state of a family of states has the most entanglement. To answer this question it would be sufficient to find a (hopefully as simple as possible) quantity that preserves the ordering of density operators with respect to entanglement, i.e. that for two measures $E_{1}$ and $E_{2}$ and any two density operators $\rho_{1}$ and $\rho_{2}$ we have that $E_{1}\left(\rho_{1}\right)>(<) E_{1}\left(\rho_{2}\right)$ is equivalent to $E_{2}\left(\rho_{1}\right)>(<) E_{2}\left(\rho_{2}\right)$.

In this paper we will compare the entanglement of formation for two spin- $1 / 2$ particles, for which a closed analytical form is known, with a quantity which was proposed in [16] as a way to quantify the degree of entanglement of a mixed state. The basis of this 'measure of entanglement' is the Peres-Horodecki criterion for the separability of bipartite systems [16]17]. Given a state of, for example, two spin- $1 / 2$ systems one calculates the partial transpose of the density operator. The state is separable exactly if the partial transpose is again a positive operator. If, however, one of the eigenvalues of the partial transpose is negative then the state is entangled. One can now imagine that the amount of entanglement is quantified by the modulus of this negative eigenvalue, i.e. the larger it is, the larger the entanglement of the state.

It is important to check whether this measure indeed preserves the ordering of density operators as it has been used for this purpose in some publications.

In section II we will explain the entanglement of formation and subsequently we summarize some properties of the negative eigenvalue measure of entanglement. Finally, we present in section III both a numerical and an analytical comparison of the two measures of entanglement with respect to the ordering of density operators induced by them. In section IV we sum up the results of this paper.

\section{ENTANGLEMENT MEASURES}

In this section we briefly describe some entanglement measures in particular the entanglement of formation and the negative eigenvalue measure of entanglement.

There are not very many good measures of entanglement. One example is the relative entropy of entanglement [6 8 ]. In fact, it gives rise to the most restrictive upper bound on the channel capacity of the depolarizing channel 8,14]. Unfortunately, even for two spin- $1 / 2$ particles no general analytical expression has been found for it so far although many special cases can be solved analytically.

A second measure of entanglement and actually the first measure of entanglement that has been proposed for mixed states is the entanglement of formation [5]. It basically describes the amount of entanglement that needs to be shared previously in order to be able to create a particular ensemble in a given state $\rho$ by local operations. Mathematically, this means that we find that pure state ensemble that realizes the state $\rho$ and which has the smallest amount of entanglement, i.e.,

$$
E_{F}(\rho)=\min _{\rho=\sum_{i} p_{i}\left|\psi_{i}\right\rangle\left\langle\psi_{i}\right|} \sum_{i} p_{i} E_{v N}\left(\left|\psi_{i}\right\rangle\left\langle\psi_{i}\right|\right),
$$

where $\left\{\left|\psi_{i}\right\rangle\right\}$ is a set of not necessarily orthogonal pure states. The entanglement of formation is known to be larger than the relative entropy of entanglement which 
proves that in general quantum state purification methods cannot recover all the entanglement that has been invested in the creation of the quantum state [8].

A nice feature of the entanglement of formation is the fact that it can be solved analytically for a system of two spin-1/2 particles [15]. This allows for fast numerical studies as the cumbersome minimization Eq. (11) can be avoided.

The entanglement of formation can be expressed in terms of the function

$$
\mathcal{E}(C)=h\left(\frac{1+\sqrt{\left(1-C^{2}\right)}}{2}\right)
$$

where

$$
h(x)=-x \log _{2} x-(1-x) \log _{2}(1-x) .
$$

For a density operator $\rho$ one defines the spin flipped state

$$
\tilde{\rho}=\left(\rho_{y} \otimes \rho_{y}\right) \rho^{*}\left(\rho_{y} \otimes \rho_{y}\right)
$$

where the ${ }^{*}$ denotes the complex conjugate in the standard computational basis $|00\rangle,|01\rangle,|10\rangle,|11\rangle$. One then finds the entanglement of formation to be

$$
E_{F}(\rho)=\mathcal{E}(C(\rho))
$$

where the so called concurrence is defined by

$$
C(\rho)=\max \left\{0, \lambda_{1}-\lambda_{2}-\lambda_{3}-\lambda_{4}\right\}
$$

Here, $\lambda_{1}, \ldots, \lambda_{4}$ are the eigenvalues, in decreasing order, of the Hermitean matrix $R=\sqrt{\sqrt{\rho} \tilde{\rho} \sqrt{\rho}}$. For properties of this measure of entanglement the reader should consult the literature [5, 15]. It is interesting to note that since $\mathcal{E}$ as a function of the concurrence $C$ is a strictly monotonous function and maps the interval $[0,1]$ on $[0,1]$ $C$ can in fact also be regarded as a measure for entanglement.

We will now consider the negative eigenvalue of the partial transpose of a density operator as a measure of entanglement. In the next section we will then compare it to the entanglement of formation.

For two spin-1/2 particles (which form the two systems $A$ and $B$ ) any disentangled state $\rho$ can be written as the convex sum of product states

$$
\rho=\sum_{i} p_{i} \rho_{i}^{A} \otimes \rho_{i}^{B} .
$$

States which permit a representation of the form Eq. (7) are also called separable. For two spin-1/2 particles there is a simple criterion to decide whether a given state is separable or not 17, 16. One calculates the partial transpose $\rho^{T_{B}}$ of the density operator $\rho$ in the computational basis. This means that we transpose only one subsystem, either subsystem $A$ or $B$. If the resulting matrix is positive semidefinite then the density operator $\rho$ is separable; otherwise it is not. Therefore the partial transpose of an entangled state has a negative eigenvalue and the idea of the negative eigenvalue measure is to use the modulus of the negative eigenvalue to quantify the entanglement of the state $\rho$. In a mathematical form this reads as

$$
E_{N}(\rho)=\left|\min \left\{0, \lambda_{1}^{T_{B}}, \lambda_{2}^{T_{B}}, \lambda_{3}^{T_{B}}, \lambda_{4}^{T_{B}}\right\}\right|
$$

where the $\lambda_{i}^{T_{B}}$ are the eigenvalues of the partial transpose $\rho^{T_{B}}$. However, we do not know whether this way of quantifying the entanglement constitutes a proper measure of entanglement. Therefore we will investigate in the next section numerically whether the entanglement of formation and the negative eigenvalue measure are compatible from a different point of view.

\section{ORDERING INDUCED BY ENTANGLEMENT MEASURES}

We would expect that any two 'good' entanglement measures should generate the same ordering of the density operators. This means that for two entanglement measures $E_{1}$ and $E_{2}$ and any two density operators $\rho_{1}$ and $\rho_{2}$ we have that

$$
E_{1}\left(\rho_{1}\right)>E_{1}\left(\rho_{2}\right) \Leftrightarrow E_{2}\left(\rho_{1}\right)>E_{2}\left(\rho_{2}\right) .
$$

Why do we expect this relation to be true? If in one measure of entanglement $\rho_{1}$ contains more entanglement than $\rho_{2}$ then we would expect that a quantum state purification method would generate more singlets from an ensemble in state $\rho_{1}$ than ensemble $\rho_{2}$. If $E_{2}$ is also a measure of entanglement then we would expect that $\rho_{2}$ would yield more singlets than $\rho_{1}$. While this reasoning is not strict, it nevertheless indicates that Eq. (9) should be true for two 'good' measures of entanglement.

In the next two subsections we will now check whether Eq. (9) is satisfied for the entanglement of formation $E_{F}$ and the negative eigenvalue measure of entanglement $E_{N}$.

\section{A. An analytical comparison}

For some classes of density operators we can easily check analytically whether the relation Eq. (9) is true. For pure states it is sufficient to consider states of the form $|\psi\rangle=\alpha|00\rangle+\beta|11\rangle$ as it follows from the Schmidt decomposition [18]. The entanglement of formation $E_{F}$ then reduces to the von Neumann entropy of entanglement while the negative eigenvalue measure yields

$$
E_{N}(|\psi\rangle\langle\psi|)=\alpha \beta=\alpha \sqrt{1-\alpha^{2}}
$$

Both measures decrease monotonically with $\alpha$ so that for pure states Eq. (9) is satisfied. It should be mentioned 
that for pure states $|\psi\rangle\langle\psi|$ with $|\psi\rangle=\alpha|00\rangle+\beta|11\rangle$ the concurrence $C$ is given by

$$
C(|\psi\rangle\langle\psi|)=2 \alpha \sqrt{1-\alpha^{2}}
$$

hence, also for arbitrary pure states $\rho=|\psi\rangle\langle\psi|$ the negative eigenvalue measure and the concurrence are connected by the simple equation

$$
C(\rho)=2 E_{N}(\rho) .
$$

Werner states are defined as

$$
\rho_{F}=\frac{4 F-1}{3}\left|\psi^{-1}\right\rangle\left\langle\psi^{-1}\right|+\frac{1-F}{3} \mathbf{1},
$$

where $|\psi\rangle=(|01\rangle-|10\rangle) / \sqrt{2}$ is the singlet state and $F \in[1 / 4,1]$. The concurrence of Werner states is given by $C\left(\rho_{F}\right)=2 F-1$ for $F \geq 1 / 2$ (otherwise $\rho_{F}$ is a separable state) while the entanglement of formation is found to be

$$
E_{F}\left(\rho_{F}\right)=-\mu \log _{2} \mu-(1-\mu) \log _{2}(1-\mu)
$$

with $\mu=1 / 2+\sqrt{F(1-F)}$. The negative eigenvalue measure simply gives

$$
E_{N}\left(\rho_{F}\right)=F-\frac{1}{2}
$$

which means that Eq. (12) is also valid for Werner states. Again, both measures decrease monotonically with $F$ and therefore Eq. (9) is satisfied.

Moreover, no counterexample to Eq. (9) can be constructed from pure states and Werner states, i.e. no two density operators $\rho_{1}$ and $\rho_{2}$ can be found which violate Eq. (9) when $\rho_{1}$ is assumed to be a pure state and $\rho_{2}$ corresponds to a Werner state. Because of the monotony of $\mathcal{E}$ in Eq. (2) this can already be seen when comparing the concurrences of $\rho_{1}$ and $\rho_{2}$ with the negative eigenvalue measure of the respective states: from Eq. (12) it then immediately follows that

$$
C\left(\rho_{1}\right)>C\left(\rho_{2}\right) \wedge E_{N}\left(\rho_{1}\right)<E_{N}\left(\rho_{2}\right)
$$

cannot be fulfilled for any value of $F$.

One may therefore suspect that Eq. (9) also holds for arbitrary mixed states. To tackle the question whether Eq. (9) is generally satisfied and hence the two entanglement measures induce the same ordering we have employed Monte Carlo simulations. If Eq. (9) is violated at all, it is - roughly speaking - also of interest to investigate how 'badly' it is violated after all. In the following subsection we present the results of the numerical test that we have performed.

\section{B. A numerical comparison}

In order to test the ordering induced by the two entanglement measures under consideration we have numerically generated a million pairs of random density matrices with respect to a certain well defined distribution. In the case of two spin- $1 / 2$ particles, which is the case of interest in this paper, one has to generate random density operators - i.e. linear, self adjoint, positive semidefinite operators $\rho$ of finite trace $(=1)$ - acting on the four dimensional Hilbert space isomorphic to $\mathbb{C}^{2} \otimes \mathbb{C}^{2}$. One can represent $\rho$ by the decomposition

$$
\rho=\sum_{i=1}^{4} p_{i}\left|\psi_{i}\right\rangle\left\langle\psi_{i}\right|
$$

with pairwise orthogonal projections, where $p_{i} \geq 0$ and $\sum_{i} p_{i}=1$. This in turn corresponds to a $4 \times 4$ matrix of the form

$$
\rho=U D U^{\dagger},
$$

where the diagonal matrix $D$ is defined by $D_{i j}=\delta_{i j} p_{i}$.

A plausible choice for the ensemble of unitary $4 \times 4$ matrices which can be used to construct density matrices according to Eq. (18) is the one with the normalized Haar measure on the group of unitary matrices $U(4)$ which is called the circular unitary ensemble 19]. It has been shown in [20] that random unitary matrices representative for the circular unitary ensemble can be obtained as follows: Let us set

$$
\begin{aligned}
U & =U^{(1,2)}\left(\phi_{12}, \psi_{12}, \chi_{12}\right) \\
& \times U^{(2,3)}\left(\phi_{23}, \psi_{23}, 0\right) U^{(1,3)}\left(\phi_{13}, \psi_{13}, \chi_{13}\right) \\
& \times U^{(3,4)}\left(\phi_{34}, \psi_{34}, 0\right) U^{(2,4)}\left(\phi_{24}, \psi_{24}, 0\right) U^{(1,4)}\left(\phi_{14}, \psi_{14}, \chi_{14}\right),
\end{aligned}
$$

where the complex $4 \times 4$ matrices $U^{(i, j)}, i, j=1, \ldots, 4$, with three real parameters $\phi, \chi$, and $\psi$ are given by

$$
U_{k l}^{(i, j)}(\phi, \psi, \chi)= \begin{cases}1, & k=l, k \neq i, j, \\ \sin \phi e^{i \chi}, & k=i, l=j, \\ \cos \phi e^{i \psi}, & k=l=i, \\ \cos \phi e^{-i \psi}, & k=l=j, \\ -\sin \phi e^{-i \chi}, & k=j, l=i, \\ 0, & \text { otherwise. }\end{cases}
$$

We now take $\psi_{i j}$ and $\chi_{i j}$ to be independent random variables with a uniform distribution in the interval $[0,2 \pi)$, and for a given random number $\xi$ distributed uniformly in the interval $[0,1)$ we choose $\phi_{i j}$ to be $\arcsin \left(\xi^{1 /(2 i)}\right)$ for $i=1, \ldots, 3$. Matrices generated in this way are then random unitary matrices of the wanted type (except for a random phase which is of no significance for our purposes). Finally, a set of random density matrices can be obtained by appropriately choosing the diagonal matrices $D$. More precisely, the random vector with entries $p_{1}, \ldots, p_{4}$ should be uniformly distributed on the manifold defined by $\sum_{i} p_{i}=1$. According to 21] this can be achieved, e.g., by setting $p_{1}=1-\xi_{1}^{1 / 3}$, 
$p_{2}=\left(1-\xi_{2}^{1 / 2}\right)\left(1-p_{1}\right), p_{3}=\left(1-\xi_{3}\right)\left(1-p_{1}-p_{2}\right)$, and $p_{4}=1-p_{1}-p_{2}-p_{3}$, where again, $\xi_{1}, \xi_{2}$, and $\xi_{3}$ are random numbers drawn with respect to a uniform distribution in the interval $[0,1)$.

To investigate whether Eq. (9) is satisfied we now draw random density matrices from the previously described ensemble and calculate the eigenvalues of the partial transposes and the eigenvalues of the products of the respective density operators with the spin flipped states. After discarding those density matrices which correspond to separable states we then determine values for $E_{N}$ and $E_{F}$ as described in section II. Finally, we check for pairs $\left(\rho_{1}, \rho_{2}\right)$ whether the sign of

$$
\Delta E_{F}\left(\rho_{1}, \rho_{2}\right)=\frac{E_{F}\left(\rho_{1}\right)-E_{F}\left(\rho_{2}\right)}{E_{F}\left(\rho_{1}\right)+E_{F}\left(\rho_{2}\right)}
$$

and

$$
\Delta E_{N}\left(\rho_{1}, \rho_{2}\right)=\frac{E_{N}\left(\rho_{1}\right)-E_{N}\left(\rho_{2}\right)}{E_{N}\left(\rho_{1}\right)+E_{N}\left(\rho_{2}\right)}
$$

is identical. Fig. 11 shows a diagram in which $\Delta E_{F}\left(\rho_{1}, \rho_{2}\right)$ is plotted versus $\Delta E_{N}\left(\rho_{1}, \rho_{2}\right)$ for $10^{4}$ pairs $\left(\rho_{1}, \rho_{2}\right)$ of random density matrices. Although there is obviously a certain correlation between $\Delta E_{F}$ and $\Delta E_{N}$, there are dots in the second and the fourth quadrant of the diagram which are associated with pairs of states which do not satisfy Eq. (9). One can therefore conclude that in the case of two spin- $1 / 2$ systems this relation is not satisfied for arbitrary mixed states, and hence, the ordering induced by the entanglement of formation $E_{F}$ and the one induced by the negative eigenvalue measure $E_{N}$ is not the same.

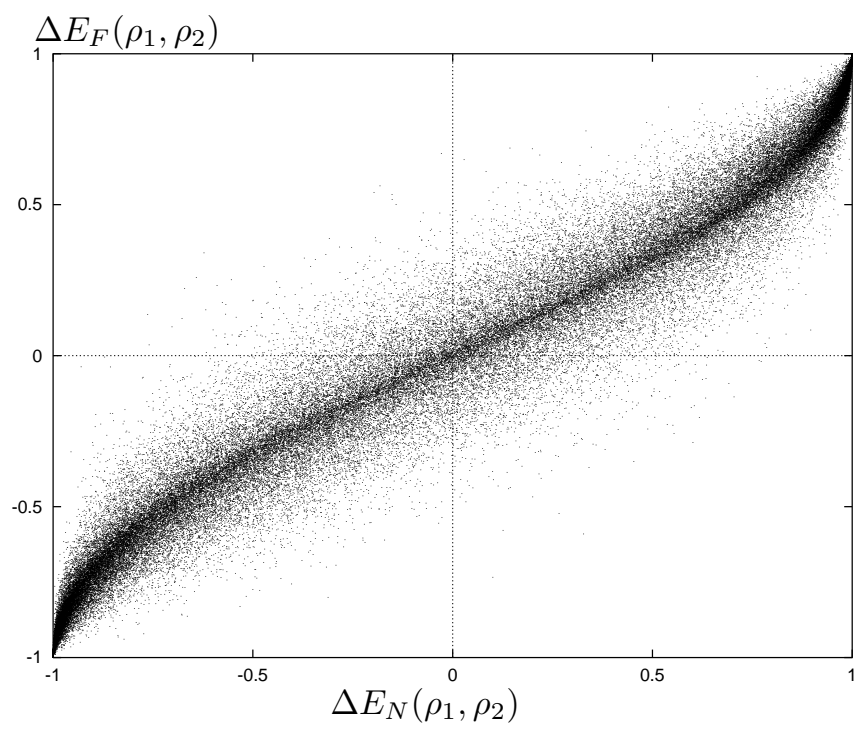

FIG. 1. $\Delta E_{F}\left(\rho_{1}, \rho_{2}\right)$ versus $\Delta E_{N}\left(\rho_{1}, \rho_{2}\right)$ for $10^{4}$ pairs of entangled states $\left(\rho_{1}, \rho_{2}\right)$. Pairs of states with $\Delta E_{F}\left(\rho_{1}, \rho_{2}\right) \Delta E_{N}\left(\rho_{1}, \rho_{2}\right)<0$ are represented by dots in the second and fourth quadrant.
It is interesting to note that most of the randomly drawn density operators do not correspond to entangled but to separable states; so more density matrices have to be discarded than can be kept for the investigation. In fact, from the numerical simulations we can estimate the probability $P_{E}$ that a created mixed state is entangled as $P_{E} \approx 0.365 \pm 0.001$, which is in complete agreement with the analytical and numerical findings presented in [21].

From the Monte Carlo simulation we can also give a rather accurate estimate of how likely it is to find a pair of states which violates Eq. (9), given that both states are entangled. From the relative frequency of a violation for a million pairs of density matrices we obtain for the estimate of this probability $P_{V}$

$$
P_{V} \approx 0.047 \pm 0.001
$$

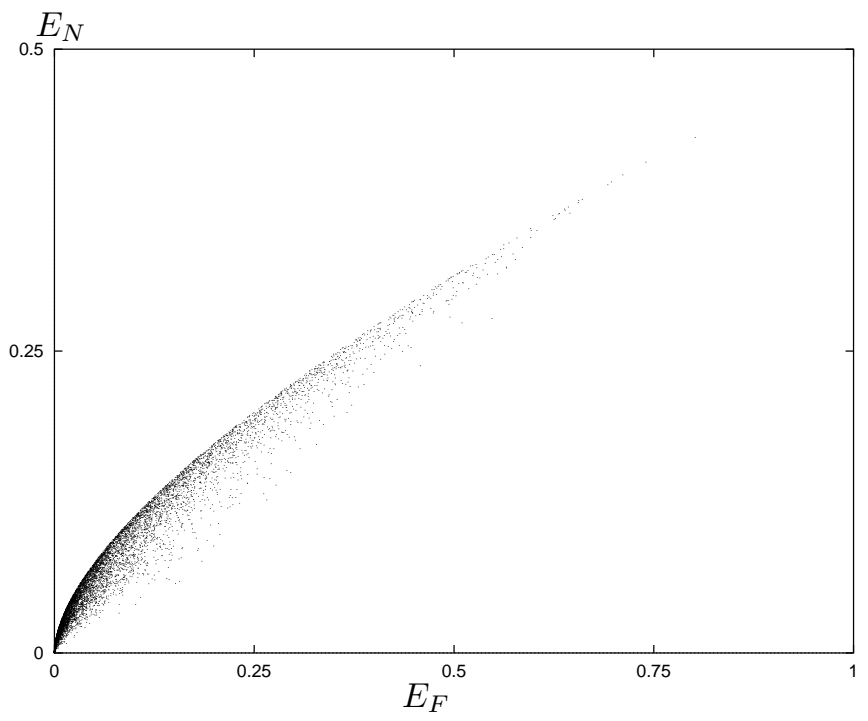

FIG. 2. Distribution of states: $E_{N}$ versus $E_{F}$.

Now that we know that the ordering is different it is interesting to see to what extent the values of $E_{N}(\rho)$ and $E_{F}(\rho)$ differ for a given state $\rho$, or how these random states are distributed in a diagram where $E_{F}$ is plotted versus $E_{N}$. Fig. 2 shows such a plot. Since the ordering induced by $E_{F}$ and $E_{N}$ is not the same, there is no strictly monotonous function $f:[0,1] \rightarrow[0,1 / 2]$ such that $E_{N}(\rho)=f\left(E_{F}(\rho)\right)$ for all states $\rho$. This is also obvious from Fig. 2. In Fig. 3 again the distribution of states is shown, but this time the negative eigenvalue measure is plotted versus the concurrence $C$. We can see that most of the dots are located close to the diagonal connecting $(0,0)$ and $(1 / 2,1)$; this diagonal corresponds to states satisfying Eq. (12). Note that the numerical simulation also strongly suggests that for a state $\rho$ with a certain value of $C(\rho)$ the upper bound for the possible values of the negative eigenvalue measure $E_{N}(\rho)$ is given by $C(\rho) / 2$, that is, that in general $C(\rho) \geq 2 E_{N}(\rho)$ holds for any state $\rho$. 
Furthermore, since for pure states the ordering induced by the two entanglement measures is the same but in general it is not, it is of interest to investigate the dependence of the probability that Eq. (9) is violated for a pair of entangled states $\left(\rho_{1}, \rho_{2}\right)$ on the 'purity of those states' with respect to a certain characterization of the purity. In Fig. 14 we show the relative number of pairs $\left(\rho_{1}, \rho_{2}\right)$ of states which do not satisfy Eq. (9) versus $S=S_{1}+S_{2}$ based on a million pairs of entangled states. Here, $S_{1}$ and $S_{2}$ are the linear entropies

$$
S_{i}=\operatorname{tr}\left(\rho_{i}-\rho_{i}^{2}\right), \quad i=1,2,
$$

of $\rho_{1}$ and $\rho_{2}$, respectively, as commonly employed, e.g., in decoherence studies 22]. Obviously, pure states correspond to a vanishing linear entropy.

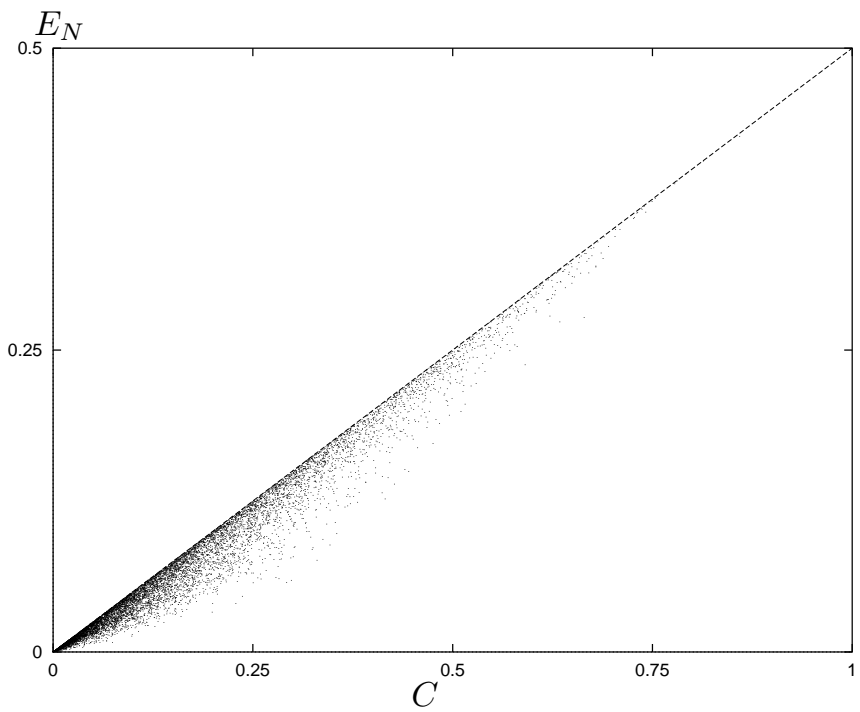

FIG. 3. Same as Fig. 月 with $E_{N}$ versus $C$.

We observe that the fraction of pairs $\left(\rho_{1}, \rho_{2}\right)$ of states with $\Delta E_{N}\left(\rho_{1}, \rho_{2}\right) \Delta E_{F}\left(\rho_{1}, \rho_{2}\right)<0$ increases monotonically with the sum of the linear entropies of the respective states. This indicates that the more mixed the two states are with respect to the arithmetic mean of their linear entropies, the larger is the probability that this pair violates Eq. (9). Above a certain cut off no pairs of entangles states can be found at all - the numerical data shown in Fig. 1 give an estimate for the probability density of finding a pair of entangled states with a certain value of $S$ (compare also [21]).

It should finally be mentioned that in [21] yet another measure of entanglement incorporating the partial transpose is proposed: For a given state $\rho$ the quantity $E=\sum_{i=1}^{4}\left|\lambda_{i}^{T_{B}}\right|-1$ is taken as a measure, where again, the $\lambda_{i}^{T_{B}}$ denote the eigenvalues of the partial transpose $\rho^{T_{B}}$ of $\rho$. While the actual value of $E$ for a given state is of course different from that of $E_{N}$, from numerical simulations we come to the same conclusion that the ordering induced by this measure is different from the one induced by the entanglement of formation $E_{F}$.

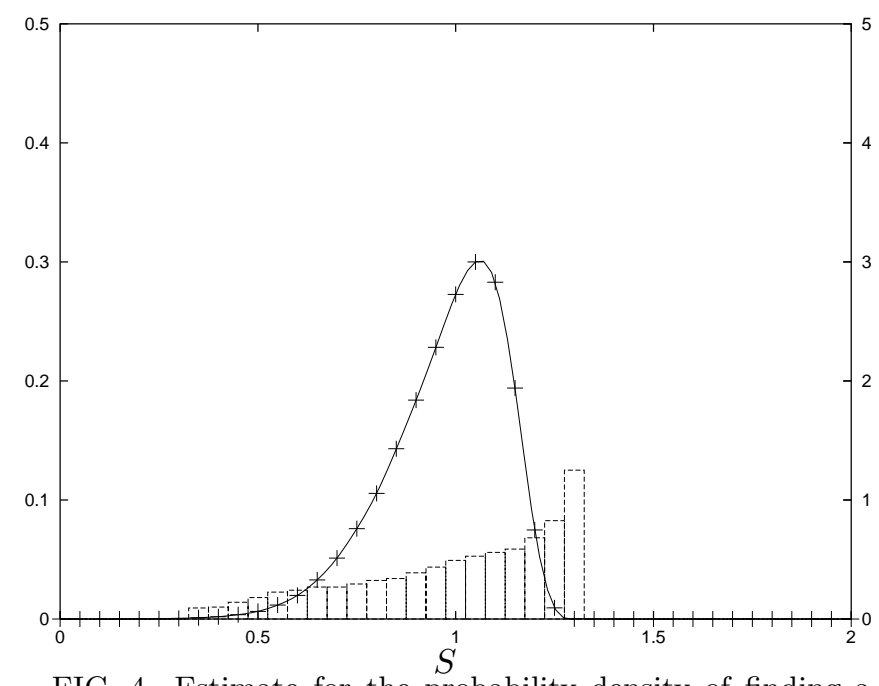

FIG. 4. Estimate for the probability density of finding a pair of states $\left(\rho_{1}, \rho_{2}\right)$ with a certain value of $S=S_{1}+S_{2}$ $(+)$. The solid line is a cubic spline interpolation. Apparently, above a certain cut off there are no pairs of entangled states any more. The histogram shows the relative number of pairs violating Eq. (9) compared to the total number of pairs of entangled states for a given value of $S$. Note that the scaling is different for both plots: the right axis belongs to the estimate for the probability density $(+)$, the left one to the relative number of violating pairs (histogram).

\section{SUMMARY}

We have compared the entanglement of formation with a potential measure of entanglement that is given by the negative eigenvalue of the partial transpose of the density operator of the system. In particular the ordering of density operators with respect to the amount of entanglement induced by the two measures has been compared both numerically and analytically. We have shown that the negative eigenvalue measure does not induce the same ordering as the entanglement of formation. Therefore we do not expect it to be a 'good' measure of entanglement. In particular it cannot, in general, be used to determine the most entangled state for a given family of density operators as it has been used previously.

\section{ACKNOWLEDGEMENTS}

The authors would like to thank Peter Knight, Vlatko Vedral, and Martin Wilkens for discussions and useful hints. This work was supported in part by the EPSRC, the European TMR Research Network ERBFMRXCT960066 and the European TMR Research Network ERBFMRXCT960087. 
[1] M.B. Plenio and V. Vedral, to appear in Cont. Physics. September 1998, also available as lanl e-print quant$\mathrm{ph} / 9804075$.

[2] C.H. Bennett, G. Brassard, C. Crepeau, R. Jozsa, A. Peres, and W.K. Wootters, Phys. Rev. Lett. 70, 1895 (1993).

[3] D. Boschi, S. Branca, F. DeMartini, L. Hardy, and S. Popescu, Phys. Rev. Lett. 80, 1121 (1998).

[4] D. Bouwmeester, J.W. Pan, K. Mattle, M. Eibl, H. Weinfurter, and A. Zeilinger, Nature 390, 575 (1997).

[5] C.H. Bennett, D.P. DiVincenzo, J.A. Smolin, and W.K. Wootters, Phys. Rev. A 54, 3824 (1996).

[6] V. Vedral, M.B. Plenio, M.A. Rippin, and P.L. Knight, Phys. Rev. Lett. 78, 2275 (1997).

[7] V. Vedral, M.B. Plenio, K. Jacobs, and P.L. Knight, Phys. Rev. A 56, 4452 (1997).

[8] V. Vedral and M.B. Plenio, Phys. Rev. A 57, 1619 (1998).

[9] C.H. Bennett, H.J. Bernstein, S. Popescu, and B. Schumacher, Phys. Rev. A 53, 2046 (1996).

[10] C.H. Bennett, G. Brassard, S. Popescu, B. Schumacher, J.A. Smolin, and W.K. Wootters, Phys. Rev. Lett. 76,
722 (1996).

[11] N. Gisin, Phys. Lett. 210, 151 (1996).

[12] M. Horodecki, P. Horodecki, R. Horodecki, Phys. Rev. Lett. 78, 574 (1997).

[13] M. Murao, M.B. Plenio, S. Popescu, V. Vedral, and P.L. Knight, Phys. Rev. A 57, R4075 (1998).

[14] E. Rains, lanl e-print quant-ph/9707002.

[15] W.K. Wootters, Phys. Rev. Lett. 80, 2245 (1998).

[16] M. Horodecki, P. Horodecki, R. Horodecki, Phys. Lett. A 223, 1 (1996).

[17] A. Peres, Phys. Rev. Lett. 77, 1413 (1996).

[18] E. Schmidt, Math. Ann. 63, 433 (1907).

[19] For a recent analysis of measures on the set of mixed states see P.B. Slater, lanl e-print quant-ph/9806039.

[20] K. Życzkowski and M. Kuś, J. Phys. A 27, 4235 (1994); see also M. Poźniak, K. Życzkowski, and M. Kuś, J. Phys. A 31, 1059 (1998).

[21] K. Życzkowski, P. Horodecki, A. Sanpera, and M. Lewenstein, lanl e-print quant-ph/9804024.

[22] W.H. Zurek, S. Habib, J.P. Paz, Phys. Rev. Lett. 70, 1187 (1993). 